\title{
Practice of the Treatment of Milk Allergy in the UK: A National Audit
}

\author{
David Luyt $^{\mathrm{a}} \quad$ M. Thirumala Krishnan ${ }^{\mathrm{b}}$ Pia Huber ${ }^{\mathrm{c}}$ Andrew Clark ${ }^{\mathrm{d}}$ \\ a University Hospitals of Leicester NHS Trust, Leicester, ${ }^{b}$ Heart of England NHS Foundation Trust and Aston \\ University, Birmingham, 'British Society for Allergy and Clinical Immunology, London, and ${ }^{\mathrm{d}}$ Cambridge University \\ Hospitals NHS Trust, Cambridge, UK
}

\section{Key Words}

Milk allergy $\cdot$ Child $\cdot$ National audit $\cdot$ Current UK practice

\begin{abstract}
Background: The management of cow's milk allergy (CMA) includes initial dairy exclusion with suitable dietary substitution, diagnostic challenges where indicated, and supervised re-introduction as the condition resolves. Information on clinician practice is limited. We audited the current practice of clinicians in the UK to evaluate adherence to international guidelines. Methods: An online questionnaire was sent to all clinicians whose practice included children identified on the national allergy society website. One hundred and sixty questionnaires were sent and 116 (73\%) responded. Results: Skin prick tests were more commonly used than serum-specific lgE as diagnostic tests. Respective proportions selecting amino acid formulas (AAF) or extensively hydrolysed formulas (EHF) in severe versus mild/moderate presentations were 78 and $40 \%$ versus 20 and $88 \%$; soya formula was the first choice in 8.6 and $24 \%$. The criteria for selection of AAF as the first choice were predominantly severe IgE-mediated and non-lgE-mediated reactions, failure to thrive and if infants refused EHF. Oral challenges might be used to diagnose CMA in $69 \%$, whereas nearly $90 \%$ performed challenges to
\end{abstract}

assess tolerance. Most of these were open challenges, more commonly with fresh milk (90\%) than baked milk (60\%). Home baked milk challenges were frequently considered (70\%), although only after consideration of the clinical scenario. Practice did not vary with experience. Conclusions: Current practice amongst UK clinicians of the management of CMA is broadly in line with current guidance, particularly the positioning of hypoallergenic milks as substitute milk choices. A notable deviation was the use of home baked milk challenges or re-introduction.

(c) 2016 S. Karger AG, Basel

\section{Introduction}

Estimated prevalences of cow's milk allergy (CMA) range from 1.8 to $7.5 \%$ of young children [1]. A UK prevalence estimate indicated that $2.3 \%$ of $1-3$-year-olds suffer from CMA [2]. Therefore, together with egg and peanut allergy, it is one of the most common food allergies in children, and as with other food allergies is thought to be increasing in prevalence $[3,4]$. CMA presents unique challenges among food allergies. The presentation varies widely, often with symptoms easily ascribed to common infant conditions like reflux or colic, with a consequent

\section{KARGER}

E-Mail karger@karger.com

www.karger.com/iaa
C 2016 S. Karger AG, Basel

1018-2438/16/1691-0062\$39.50/0
Correspondence to: Dr. David Luyt

Children's Allergy Service

University Hospitals of Leicester, Infirmary Square

Leicester LE1 5WW (UK)

E-Mail david.luyt@ @uhl-tr.nhs.uk 
delay to diagnosis [5]. In addition, affected individuals are effectively allergic to their entire diet so that a treatment plan of allergen avoidance alone is not enough, as dietary solutions of alternative milks need implementing immediately.

Clinicians and other health providers have a number of milks or drinks to choose as feed substitutes in children with CMA. Although not all of these options are appropriate substitutes based on either allergy or nutritional criteria, clinical experience suggests that inappropriate milks continue to be prescribed [6-8]. There is very little audit evidence of clinical practice [9].

Practice parameters have recently been published by the World Allergy Organisation (WAO) and the European Society of Gastroenterology, Hepatology and Nutrition (ESPGHAN), and by expert panels in Australia and Europe [1, 10-12]. However, given the unique nature of health care provision in the UK, the British Society for Allergy and Clinical Immunology (BSACI) Standards of Care Committee (www.bsaci.org.Guidelines/bsaciguidelines-and-SOCC) felt that there was a place for a UK-based guideline to enhance and harmonise practice in the UK.

The objective of developing and disseminating guidelines is to share best practice and thereby enhance care. The desired changes in care can be measured by audits of practice before and after the publication of guidelines. The BSACI Standards of Care Committee (SOCC) therefore wished to understand, prior to the publication of their proposed guideline, the practice of management of CMA in the UK.

\section{Materials and Methods}

The BSACI database carries practice information on members (doctors, nurses and allied health professionals). All members whose allergy practice incorporated children were included in this audit. An online questionnaire, developed by members of the BSACI SOCC, was sent to these 160 members in November 2013. There were 17 questions, all of which were multiple-choice and some in which respondents were advised to consider multiple responses, where deemed appropriate. The first 8 determined the allergy practice of the respondent. The remaining 9 questions interrogated the individual clinical practice of milk substitute selection and use of oral challenges (online suppl. table 1; for all online suppl. material, see www.karger.com/doi/10.1159/000444171). The initial responses were logged and two reminder e-mails were sent in December 2013 and January 2014 to maximise the response rate. The online audit was open for 8 weeks from the time of initial contact. Clinical practice was evaluated against practice demographics using specialty designation as a marker for clinical experience.

National Audit of Milk Allergy Treatment

\section{Results}

\section{Demographics of Questionnaire Respondents and} Clinical Practices

A total of $114 / 160$ (71\%) responded, of whom $26(22 \%)$ described themselves as paediatric allergists (PAs) and 56 (49\%) as paediatricians with an interest in allergy. Of the remaining 11 doctors who responded, 1 was a paediatric gastroenterologist, 5 were general practitioners, 2 were adult allergists and 3 were trainees. In addition, responses were received from 12 nurses and 9 dietitians. Two thirds of respondents described their main continuing professional development area as allergy. Not all respondents replied to all questions (online suppl. table 1).

The practice settings were described as a dedicated paediatric allergy clinic by $88(76 \%)$ respondents and as a general paediatric clinic by 37 (32\%). Other settings included a dedicated paediatric gastroenterology clinic (3), combined adult and paediatric allergy clinic (4), general practice (3) and a dietetic clinic (7). Some respondents worked in more than one clinical setting. Service activity, as measured by weekly scheduled clinics and annual new patient numbers, varied widely: $27 \%$ have less than one weekly clinic and $53 \%$ conduct 2 or more clinics a week, whilst $25 \%$ of services see less than 200 new patients a year, $43 \%$ see $200-500$ and $32 \%$ see over 500 . Clinicians were present in $91 \%$, dietitians in $59 \%$ and specialist nurses in $56 \%$ of clinics during a routine consultation.

The frequencies with which tests were used to investigate for CMA as routine clinical practice are shown in figure 1. Skin prick tests (SPTs) with commercial reagents were used most commonly (by $84 \%$ of respondents), whilst nearly half of respondents also used fresh cow's milk (CM). Blood tests assaying specific IgE to whole CM were also used frequently (72\%), although less so than SPTs. Fifty-three percent used both SPTs and specific IgE. A sizable number of respondents (1 in 5) included serum assays of CM components in their routine practice.

\section{Alternative Milk Selection Practices}

Alternative milk selection practices were assessed using a scenario of an immediate allergic reaction presenting with either severe/anaphylactic or mild to moderate symptoms, and by criteria selection of an amino acid formula (AAF) over an extensively hydrolysed formula (EHF).

When clinicians were faced with the clinical presentation of a severely allergic child who experienced symptoms whilst exclusively breast-fed (i.e. allergic reactions to trace amounts of CM in maternal breast milk) and who

Int Arch Allergy Immunol 2016;169:62-68 
Fig. 1. Question 8: As part of a routine consultation in your clinic, which allergy tests do you use to investigate CMA? (Please tick all that apply.)

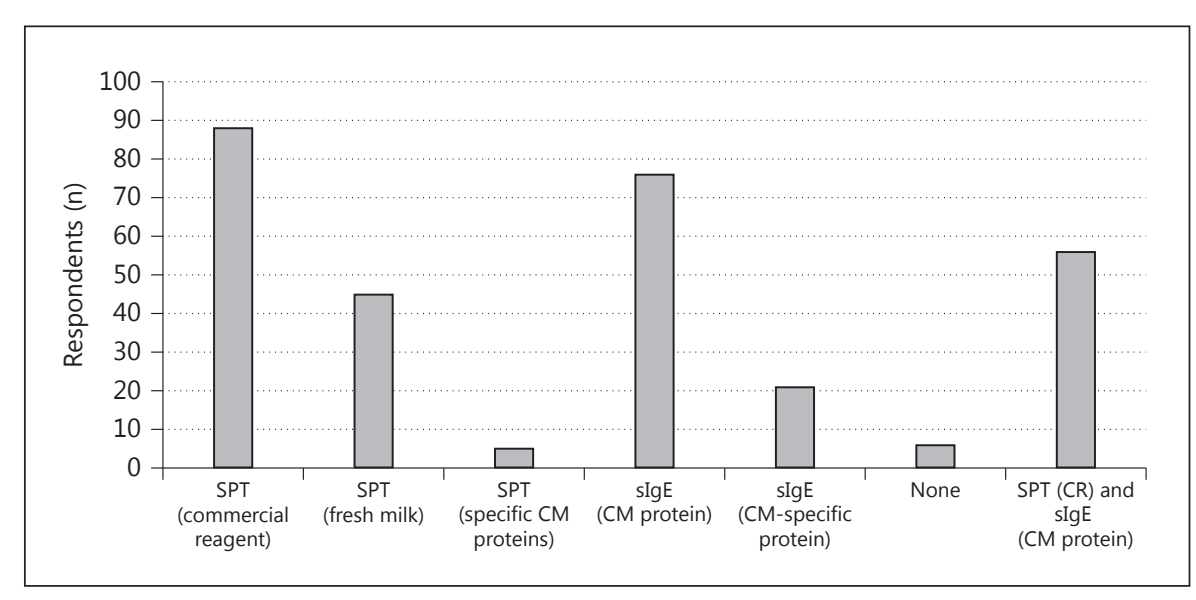

experienced an anaphylactic reaction on first $\mathrm{CM}$ contact per se (scenario 1), 78\% selected an AAF, while half again (40\%) would select an EHF. A smaller proportion chose a soya formula (8.6\%), 2 selected a nut or cereal drink and 2 chose goat's milk. By contrast, when faced with a less sensitive child who experienced mild to moderate symptoms with CM exposure (scenario 2), the majority (88\%) select EHF as the milk of choice, a quarter would offer a soya formula and 1 in 5 an AAF. Again 2 selected a nut or cereal formula but none chose goat's milk (fig. 2).

When considering criteria for selecting an AAF over an EHF, three quarters indicated both IgE-mediated (anaphylaxis) and non-IgE-mediated (food protein-induced enterocolitis syndrome) severe reactions as reasons for choosing an AAF, and half would use this formula when the child was failing to thrive or EHF was refused. A child with multiple allergies was also regarded as a reason for selecting an AAF, although less so than the other options (fig. 3).

\section{Use of Diagnostic or Monitoring Food Challenges}

Sixty-nine percent might use oral challenges to diagnose CMA, whereas nearly $90 \%$ performed oral challenges to assess tolerance to CMA. All but 1 used open oral challenges, and in addition 15\% used double-blind challenges to diagnose and $8 \%$ to monitor tolerance (fig. 4). When performing challenges, $60 \%$ used baked milk and $90 \%$ fresh milk. When considering where respondents would advise baked milk challenges to be performed, $16 \%$ suggested at home only, $14 \%$ in hospital only and $70 \%$ at home or in hospital based on clinical assessment. Home baked milk challenges were therefore recommended as part of care for CMA by over $80 \%$ of clinicians working with children with CMA.

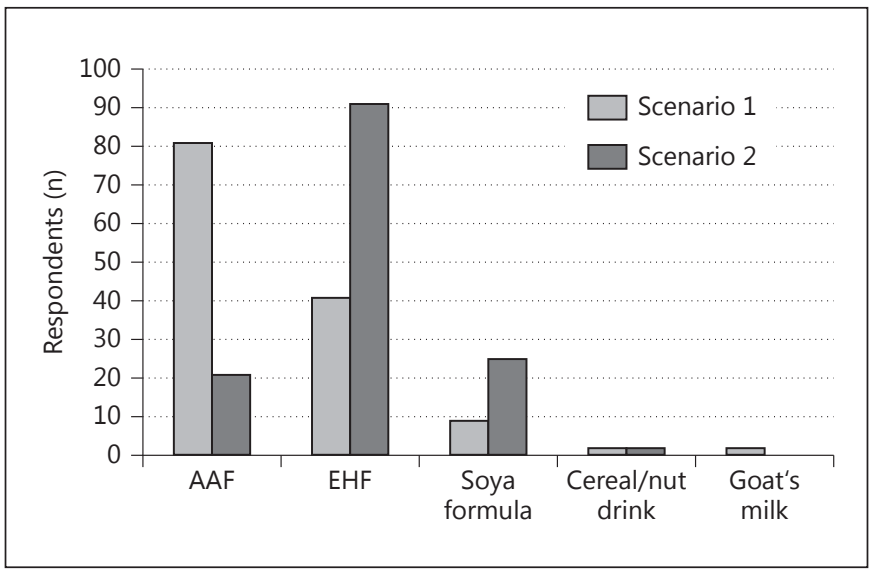

Fig. 2. Alternative milk selection practices according to the severity of presentation of CMA. Clinical scenario 1 (question 9): You are presented with a 6-month-old female infant who you diagnose with CMA. She has been exclusively breast-fed. Her mother has dairy in her diet. She has been difficult to feed, frequently vomits and has loose stools. When weaned she had an allergic reaction to baby rice containing milk powder with an urticarial rash, profuse vomiting, pallor and drowsiness. Clinical scenario 2 (question 10): You are presented with a 6-month-old female infant who you diagnose with CMA. She has been exclusively breast-fed. Her mother has dairy in her diet. She has been a well, thriving, contented baby. When weaned she had an allergic reaction to baby rice containing milk powder with an urticarial rash and mild vomiting only.

\section{Association of Clinical Behaviour and Practice Demographics}

Alternative milk selection and food challenge practices were assessed against practice demographics. Respondents were divided into 3 groups according to their response to question 1 , in which they were asked to describe their specialty: PAs, paediatricians with an interest in al- 
Fig. 3. Alternative milk selection practices according to clinical criteria. Question 11: When evaluating an infant with diagnosed CMA for prescription of a substitute formula, which of the following criteria would prompt you to select an amino acid formula over an extensively hydrolysed formula? (You may tick more than one.) FPIES = Food protein-induced enterocolitis syndrome.

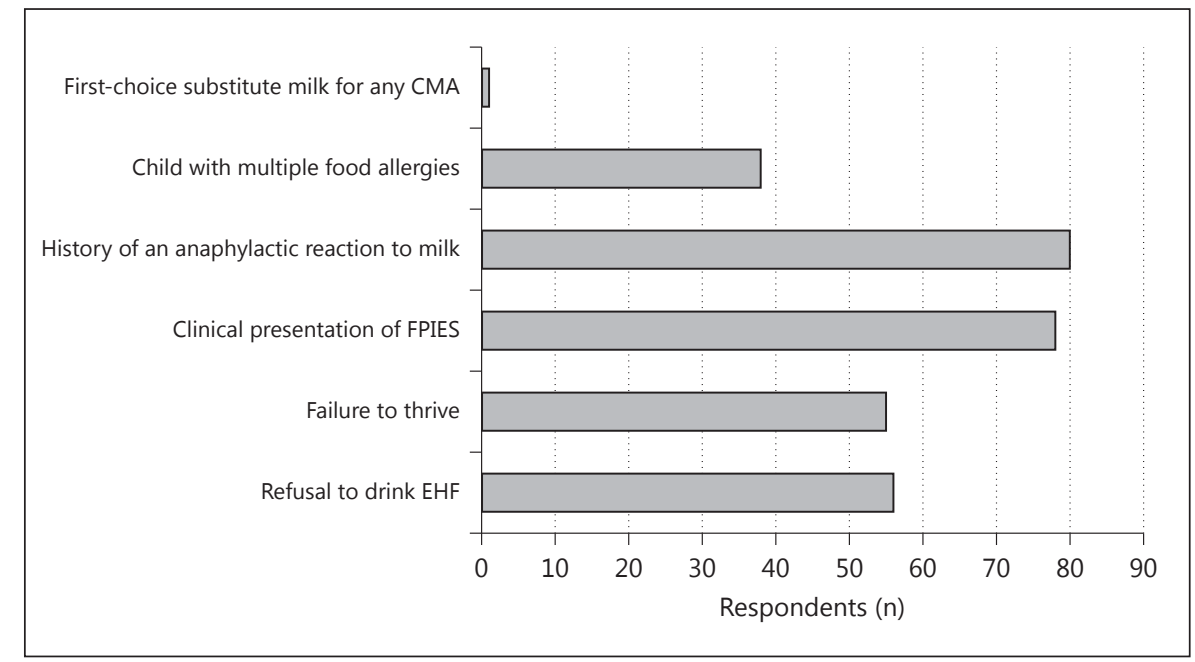

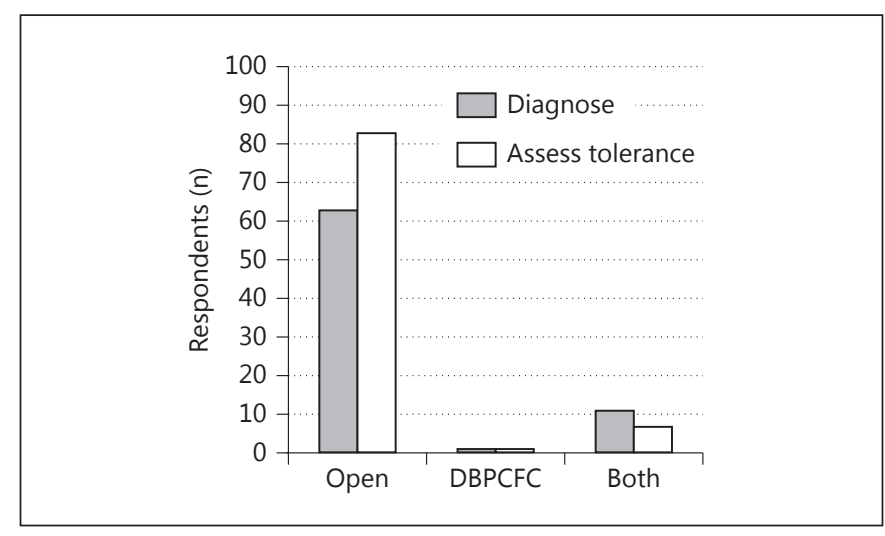

Fig. 4. Use of diagnostic or monitoring food challenges. Questions 12-15: Does your service perform oral challenges to diagnose CMA or assess tolerance in children with CMA? If you ticked yes, are the oral challenges open challenges, double-blind placebo-controlled food challenges (DBPCFC) or both?

lergy (PIAs), and the remainder. We used this practice type as a measure of clinical experience as $83 \%$ of PAs reported more than 500 new patient consultations a year compared with only $9 \%$ of PIAs.

There were no differences in practice in the three groups. Where alternative milk selection was assessed, the proportions in each group choosing alternative milks did not differ with the possible exception that infant soya formulas tended to be more frequently selected by PAs in the first scenario of a 6-month-old child who was extremely sensitive to CM (23 vs. 5\% for PIA). Use of diagnostic challenges did not differ in the practices of PAs and
PIAs, although more PAs tended to use challenges to diagnose CMA ( 81 vs. $66 \%$ for PIAs). This may reflect the complexity of presentation to these clinics. Reintroduction practices did not differ.

\section{Discussion}

This is the first national audit of the management, including diagnostic tests and treatment strategies, of CMA performed prior to the publication of a national guideline. The clinician questionnaire methodology provides unique information. Previous studies reported on much smaller cohorts or derived information on the prescription of alternative milk from analyses of prescription data $[9,13]$.

CMA is distinct from other food allergies as at presentation the affected infant is usually effectively allergic to his or her entire diet. The standard dietary strategy in food allergies of avoidance needs to be supported with the prescription of an alternative suitable replacement milk. Furthermore, most children with CMA will 'grow out' of their allergy in early childhood, so unlike persistent allergies such as peanut or fish allergy, management should also include an early CM re-introduction plan [4]. There is evidence that this may benefit the individual by accelerating the resolution of CMA [14]. Thus, as the management of CMA needs to address these two issues, we included corresponding questions in our audit.

\section{Alternative Milk Selection Practices}

We used two clinical scenarios to assess the treatment preference of our cohort of respondents regarding the 
choice of alternative milk selection guided by the condition it addresses. The first of these scenarios portrays a presentation of CMA where a 6-month-old child is extremely sensitive as she reacts to CM protein in maternal breast milk whilst exclusively breast-fed, and experiences an anaphylactic reaction with pallor and drowsiness with contact to CM per se. In the second scenario, the child is less sensitive as she does not respond to CM protein in maternal milk and her symptoms with CM exposure are mild. The questions offered three milks that are suitable for CMA (AAF, EHF and soya) and two that are regarded as unsuitable (cereal- or nut-based drinks and goat's milk) $[7,8]$. Any of the suitable substitutes can be used in either scenario. However, the risk of failure of EHF is up to $10 \%$ in all infants with CMA, and possibly higher in the presence of severe enteropathy or with multiple food allergies [15]. In addition, with the exception of the small percentage that reacts to EHF, there is no clear evidence that AAF is a more suitable substitute formula in CMA. As AAF is more expensive than EHF, expert panels do not support the preferential use of AAF over EHF except in those who do not tolerate $\operatorname{EHF}[1,10,11]$.

Alternative hypoallergenic (EHF or AAF) milk selections by respondents reflected consideration of these issues as about $80 \%$ selected AAF for the severe presentation and $90 \%$ chose EHF for the milder presentation. However, $40 \%$ selected EHF for the severe presentation where the risk of failure is higher; 2 respondents qualified their selection with comments that an EHF would initially be tried to determine tolerance and if that failed an AAF would be offered. This practice is thus generally consistent with the recommendations of expert panels $[1,10$, 11]. It is also possible that AAF selection is limited by the restriction of prescriptions in certain institutions because of their cost.

Criteria identified for AAF selection were again consistent with alternative milk selection for the clinical scenarios and with published guidelines [1]. Only one respondent selected the option of 'first choice substitute for any CMA'. The proportion of respondents who selected infant soya formulas as a suitable alternative milk was low (9 and 24\% for the more severe and milder presentations, respectively), although greater than the choice of an AAF in the milder scenario (20 and 24\%). This may reflect concerns with risks of failure because of concomitant soya allergy or be influenced by previous guidance in the UK where soya is not recommended in children under 6 months of age $[6,16]$.

The UK has a National Health Service (NHS) where prescriptions for children are free. As AAF and EHF are pharmaceutical prescriptions, these milks are provided free at source to individual patients. The cost of the health care in primary care, which includes repeat prescriptions of AAF and EHF, is funded through individual general practitioner group budgets. These practitioner groups may place restrictions of prescriptions in an attempt to limit spending on high-cost prescriptions.

The hypoallergenic milks are considerably more expensive than CM infant formulas. The cost, for example, of feeding an infant aged between 3 and 12 months in the UK on AAF is approximately GBP 1,800 (equivalent to USD 2,800) compared with GBP 600 with EHF, GBP 180 with soya formulas and GBP 150 with standard CM infant formulas. As prescriptions are free, the selection of a formula as a suitable substitute milk will not be determined by whether an individual family can afford it. However, local policies may guide clinicians to less expensive formulas as soon as this is clinically possible, like changing from a hypoallergenic to a soya formula.

The contrast of selection criteria of UK clinicians with colleagues in Australia and South Africa may at least in part be based on affordability and the funding arrangements of respective health care systems. In Australia, where clinicians practise in privately funded systems not restricted by institutional protocols, AAFs seem to be used increasingly as the first-line treatment for CMA, as evidenced by the observation of a rising number of AAF prescriptions [13]. Differences were also noted from limited prescribing data used to assess the health economic impact of managing CMA in South Africa. Sixteen paediatricians working in the private health sector were interviewed. The first-choice alternative milks were soya (57\%) and EHF (38\%), and where soya was not tolerated either an EHF (62\%) or AAF (28\%) was recommended. Three paediatricians working in the public sector estimated that 93 and 7\% of CMA sufferers would be advised to be fed with soya or EHF, respectively [9]. Soya, the cheapest of the alternative formulas, was therefore the first-line choice in the least affluent health care environments.

\section{Diagnosis and Monitoring of CMA}

The diagnosis of food allergy is based on a suggestive history together with confirmatory allergy tests and food challenges [17]. In CMA, eliciting a suggestive history is pivotal in making a diagnosis as symptoms can be similar to other conditions common in infants, like reflux and colic, and because about half present with non IgE-mediated allergy where allergy tests are negative despite the presence of allergy. Nevertheless, in our cohort of respondents both SPTs and serum sIgE assays were widely used, and about half of
66

Int Arch Allergy Immunol 2016;169:62-68 DOI: $10.1159 / 000444171$
Luyt/Krishnan/Huber/Clark 
those using commercial reagents in SPTs also used fresh milk. Of interest is that one in five respondents reported using tests assaying specific CM proteins. Their use in the diagnosis of CMA still needs further clarification [18].

The indications for oral food challenges include identifying foods causing acute reactions for the initial diagnosis of food allergy and for monitoring the resolution of food allergy [19]. We determined whether oral challenges were offered by services to either diagnose or monitor resolution, and noted them to be widely available. A small number were able to perform double-blind placebo-controlled food challenges where indicated. Fresh milk is used more commonly than baked milk as challenge food.

We further determined whether baked milk challenges are performed at home or in hospital. Eighty-six percent of respondents perform home challenges. These appear to be conducted with due consideration as the selection of location was qualified by assessing risk based on the type of CMA, severity of previous reactions, level of sensitisation and parental anxiety, as well as using a strict protocol. Some respondents specified that the first dose might also be given in hospital. Introduction of the lower allergenic form of CM as baked milk at home is therefore widespread practice in paediatric allergy services in the UK. The early introduction of baked milk is an important part of the management of CMA as tolerance may accelerate CMA resolution. Home programmes will make this more widely available as limitations of hospital facilities presently limit access. However, most guidelines currently recommend that re-introductions be supervised in hospital [19]. Further audit of these home introductions, particularly patient selection criteria, introduction protocols and outcome, including adverse events, is needed to assess this clinical practice.

\section{Practice Demographics and Clinical Practice}

Data from audits of clinical practice may be used to target education for groups shown to be deviating from guidelines. The two main clinical decision groups of alternative milk selection and re-introduction that were assessed did not differ when compared between more and less experienced clinicians in this study. All but a small number of responses regarding the selection of alternative milks were appropriate.

The comparisons between clinicians in this study did not direct us to the education needs for specific groups of clinicians. This is not surprising as the majority of responses were from clinicians whose primary clinical activity is the management of children with allergies. Perhaps conducting a similar audit in a wider range of clini- cians, general paediatricians or primary care physicians might provide more useful data on education needs.

\section{Limitations of the Study}

Whilst this paper proposes to reflect the practice of clinicians treating children with milk allergy in the UK, we recognise that there are a number of limitations. The study recruited respondents from the BSACI database, meaning results would reflect clinicians who were more allergy orientated, and does not reflect the opinions and practice of the large number of general paediatricians not working in allergy clinic settings, many of whom are managing children with milk allergy. Furthermore, whilst the response rate was pleasingly high, we have no practice information on non-respondents. Nevertheless, the number of respondents in this study is considerably higher than that in other studies assessing clinician practice in the treatment of milk allergy $[9,13]$.

Further limitations relate to the breadth of questions on the management of milk allergy. The questionnaire focussed on an alternative milk selection and milk re-introduction. The investigators chose these as they deemed them highly relevant, particularly the latter to reflect changing practices in the management of milk allergy. The questionnaire did not, however, interrogate practice differences for the different types of milk allergy, for example, although current guidelines do not differentiate between IgE- and non-IgE-mediated milk allergy in advice on treatment, possibly because of a lack of evidence.

\section{Conclusion}

This audit provides an insight into the scope of the paediatric allergy services available in the UK for the management of CMA. It demonstrates that practice is broadly in line with current guidance from expert panels, in particular the positioning of the hypoallergenic milks as alternative milk choices. A notable deviation from most guidelines is the common use of home baked milk challenges (re-introduction). Further objective assessment of the safety and efficacy of unsupervised home milk re-introduction is therefore necessary to consider whether it merits inclusion in future guidelines.

\section{Disclosure Statement}

The authors declare no conflict of interest in relation to this work. This research received no specific grant from any funding agency in the public, commercial or not-for-profit sectors. 


\section{References}

1 Fiocchi A, Brozek J, Schünemann HJ, Bahna SL, von Berg A, Beyer K, Bozzola M, Bradsher J, Compalati E, Ebisawa M, Guzman MA, Li H, Heine RG, Keith P, Lack G, Landi M, Martelli A, Rancè F, Sampson H, Stein A, Terraciano L, Vieths S: World Allergy Organization (WAO) diagnosis and rationale for action against cow's milk allergy (DRACMA). World Allergy Organ J 2010;3:57-161.

2 Venter C, Pereira B, Voigt K, Grundy J, Clayton CB, Higgins B, Arshad SH, Dean T: Prevalence and cumulative incidence of food hypersensitivity in the first 3 years of life. Allergy 2008;63:354-359.

3 Lack G: Update on risk factors for food allergy. J Allergy Clin Immunol 2012;129:11871197.

4 Wood RA, Sicherer SH, Vickery BP, Jones SM, Liu AH, Fleischer DM, Henning AK, Mayer L, Burks AW, Grishin A, Stablein D, Samson HA: The natural history of milk allergy in an observational cohort. J Allergy Clin Immunol 2013;131:805-812.

5 Sladkevicius E, Nagy E, Lack G, Guest JF: Resource implications and budget impact of managing cow milk allergy in the UK. J Med Econ 2010;13:119-128.

6 Klemola T, Vanto T, Juntunen-Backman K, Kalimo K, Korpela R, Varjonen E: Allergy to soy formula and to extensively hydrolyzed whey formula in infants with cow's milk allergy: a prospective, randomized study with a follow-up to the age of 2 years. J Pediatr 2002; 140:219-224.
7 Restani P, Beretta B, Fiocchi A, Ballabio C, Galli CL: Cross-reactivity between mammalian proteins. Ann Allergy Asthma Immunol 2002;89:11-15.

8 Department of Health: Advice on infant milk based on goat's milk. London, Department of Health, 2006.

9 Sladkevicius E, Guest JF: Modelling the health economic impact of managing cow milk allergy in South Africa. J Med Econ 2010;13: 257-272.

10 Koletzko S, Niggemann B, Arato A, Dias JA, Heuschkel R, Husby S, Mearin ML, Papadopolou A, Ruemmele FM, Staiano A, Schäppi, Vandenplas Y: Diagnostic approach and management of cow's-milk protein allergy in infants and children: ESPGHAN GI Committee practical guidelines. J Pediatr Gastroenterol Nutr 2012;55:221-229.

11 Allen KJ, Davidson GP, Day AS, Hill DJ, Kemp AS, Peake JE, Prescott SL, Shugg A, Heine RG: Management of cow's milk protein allergy in infants and young children: an expert panel perspective. J Pediatr Child Health 2009; 45:481-486.

12 Vandenplas Y, Brueton M, Dupont C, Hill D, Isolauri E, Koletzko S, Oranje AP, Satiano A, Dupont C: Guidelines for the diagnosis and management of cow's milk protein allergy in infants. Arch Dis Child 2007;92:902-908.
13 Kemp A: Hypoallergenic formula prescribing practices in Australia. J Pediatr Child Health 2006;42:191-195.

14 Kim JS, Nowak-Wegrzyn A, Sicherer SH, Noone S, Moshier EL, Sampson HA: Dietary baked milk accelerates the resolution of cow's milk allergy in children. J Allergy Clin Immunol 2011;128:125-131.

15 De Boissieu D, Dupont C: Allergy to extensively hydrolysed cow's milk protein in infants: safety and duration of amino-acid formula. J Pediatr 2002;141:271-273.

16 Scientific Advisory Committee on Nutrition: Soy based infant formula. SMCN/03/10. 2003.

17 Burks AW, Tang M, Sicherer S, Muraro A, Eigenmann PA, Ebisawa M, Fiocchi A, Chiang W, Beyer K, Wood R, Hourihane J, Jones SM, Lack G, Sampson HA: ICON: food allergy. J Allergy Clin Immunol 2009;129:906-920.

18 D’Urbano LE, Pellegrino K, Artesani MC, Donnanno S, Luciano R, Riccardi C, Torzi AE, Rava L, De Benedetti F, Cavagni G: Performance of a component-based allergen-microarray in the diagnosis of cow's milk and hen's egg allergy. Clin Exp Allergy 2010;40: 1561-1570.

19 Nowak-Wegrzyn A, Assa'ad AH, Bahna SL, Bock A, Sicherer SH, Teuber SS: Work Group report: oral food challenge testing. J Allergy Clin Immunol 2009;123:S365-S383. 